\title{
CONVERGENCE PROPERTIES OF ANALYTICAL TARGET CASCADING
}

\author{
Nestor Michelena* Hyungju Park ${ }^{\dagger}$ Panos Papalambros*
}

\begin{abstract}
Analytical target cascading (ATC) is a relatively new methodology for the design of engineering systems. ATC deals with the issue of propagating desirable top level product design specifications (or targets) to appropriate targets at lower levels in a consistent and efficient manner. Most existing problem formulations for multilevel design often exhibit convergence difficulties. In this article, it is proved that under convexity assumptions the ATC process converges to the optimal solution of the original design target problem.
\end{abstract}

\section{INTRODUCTION}

In a typical product development process one of the early steps is the verification that the resulting product or system will meet some predefined design specifications or targets T. Assuming that analytical or computational capabilities exist to compute the responses $\mathbf{R}$ of the system for a given design $\mathbf{x}$, the $d e-$ sign target problem can be formulated as the mathematical optimization problem

$$
\begin{array}{r}
\min _{\mathbf{x}}\|\mathbf{R}(\mathbf{x})-\mathbf{T}\| \\
\text { subject to } \mathbf{g}(\mathbf{x}) \leq \mathbf{0} \text { and } \mathbf{h}(\mathbf{x})=\mathbf{0},
\end{array}
$$

where $\mathbf{g}$ and $\mathbf{h}$ are design constraint functions.

For a complex product, such as an automobile or aircraft, direct solution of Problem (1) is not possible. Instead, the overall product targets must be translated to proper targets for the various parts that constitute the product, which are complex products themselves and must be designed in a relatively independent manner. Thus, the design target problem becomes one of propagating (or "cascading") targets throughout a hierarchy representing the decomposition of the product into its parts. The difficulty is that complex product parts are never really independent of each other, so the targets set for

\footnotetext{
${ }^{*}$ Department of Mechanical Engineering, University of Michigan, Ann Arbor, MI 48109. e-mail: \{nestorm, pyp\}@umich.edu

${ }^{\dagger}$ Department of Mathematics and Statistics, Oakland University, Rochester, MI 48309. e-mail: park@oakland.edu.
}

them must be consistent with each other. Moreover, there must be an assurance that if the individual part targets are met, then the overall target for the entire product will be also met. Finally, recognizing that this entire process is likely to be quite complicated, efficient allocation of targets at an early stage is highly desirable.

In the sequel, the main assumption made is that the performance of the product can be analyzed and adequately described by the functions $\mathbf{R}, \mathbf{g}$, and $\mathbf{h}$, and that these functions can be computed for any given design $\mathbf{x}$. Hence, the term "analytical" is used to characterize the target cascading process.

Analytical target cascading (ATC) ${ }^{1,2,3}$ is a formal methodology for multidisciplinary optimal design. First, the design target problem is partitioned into a hierarchical set of subproblems associated with the supersystem (i.e., the product itself) and the systems, subsystems, and components making up the supersystem. The formulation is general enough to account for any number of levels in this hierarchy. Design specifications (or targets) defined at the top supersystem level are then cascaded down to lower levels following the prescribed ATC process. Once lower-level targets are identified, individual design target subproblems are formulated at each level using more detailed models and complex simulations. Thus, components, subsystems, and systems can be designed to match cascaded targets in a manner consistent with the overall targets.

The main benefits of target cascading are reduction in design-cycle time, avoidance of design iterations late in the development process, and increased likelihood that physical prototypes will be closer to production quality. Target cascading also facilitates concurrency in system design: Once targets are identified for systems, subsystems, and components, the latter elements can be isolated and designed in detail independently, allowing the outsourcing of subsystems and components to suppliers.

Analytical target cascading has been applied in automotive vehicle design to cascade ride quality and handling specifications utilizing suspension, tire, 
and spring analysis models ${ }^{4}$. A recent application of $\mathrm{ATC}^{5}$ involves the design of an advanced heavy tactical truck, which has a series hybrid electric powertrain configuration, and emphasizes fuel economy, ride, and mobility characteristics. In an application to vehicle redesign ${ }^{6}$, ATC is used to cascade fuel economy, performance, and ride quality specifications to the suspension, engine, and transmission systems of a U. S. class VI commercial truck. ATC has also been extended to the design of product families with predefined platforms ${ }^{7}$ to accommodate the presence of shared systems, subsystems, or components.

Several other formulations for multilevel design of hierarchical systems have been proposed; unfortunately, they often exhibit convergence difficulties. In structural optimization, for example, it is common to take advantage of weakly-coupled local structures and to formulate the design problem as a hierarchical problem. Lower-level design subproblems are coupled only through interactions with higher levels $8,9,10,11$. One drawback of these formulations is that derivatives of the lower-level optima may be discontinuous functions of the higher-level variables, making the multilevel problem more ill-conditioned than the original problem ${ }^{12,13}$. In collaborative optimization (CO $)^{14}$ subsystem analyses are decoupled by introducing compatibility constraints at the system level after reformulating the design problem as a bilevel programming problem. A drawback of this formulation is that the system-level constraint Jacobian either vanishes at all feasible points of the system-level problem or is discontinuous at a solution $^{15}$. Moreover, the convergence behavior of optimization algorithms applied to $\mathrm{CO}$ might be erratic.

In this article, global convergence properties of the ATC formulation, when used together with optimization algorithms, are proved under convexity assumptions - hence establishing ATC as a robust formulation for multidisciplinary optimal design.

\section{AnAlytical target CASCADing AND THE DESIGN TARGET PROBLEM}

Design of a product, or supersystem, entails determining the values of design variables such that the supersystem meets its design targets. For an automotive vehicle, these targets can be measures of fuel consumption, emissions, performance, handling, ride quality, cost, and so on. ATC assumes that the supersystem and associated models can be hierarchically partitioned into systems, subsystems, and components, with as many levels as needed. Each entity at each level, which corresponds to a node of the tree structure, is called an "element." Figure 1 shows a typical decomposition of an automotive supersystem.

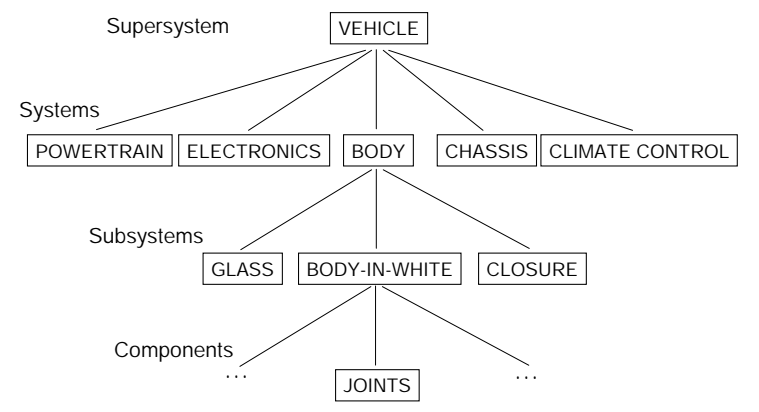

Figure 1: Typical decomposition of an automotive supersystem

\subsection{Assumptions and definitions}

The following assumptions are made with respect to the supersystem and the models describing its behavior:

- Models describing the behavior or response of each element in the problem hierarchy are available at an appropriate level of fidelity. That is, they are models of low fidelity at the top (e.g., system) levels and high fidelity at the bottom (e.g., component) levels. These models can be analytical or experimental, quantitative or qualitative. Approximate analytical models need to be generated if not available.

- The interaction between two consecutive levels in the hierarchy is similar at all levels except for the top (i.e., supersystem) and bottom (e.g., component) levels. Supersystem/system and subsystem/component interactions are special cases of the more general system/subsystem interaction of intermediate levels. This enables using similar ATC formulations for any two levels of the hierarchy.

- To represent the hierarchy of the partitioned design problem, the set $\mathcal{E}_{i}$ is defined as the collection of all elements at the $i$-th level. The supersystem level corresponds to the 0-th level, i.e., the supersystem corresponds to the element $l \in \mathcal{E}_{0}$. For each element $j$ in the set $\mathcal{E}_{i}, \mathcal{C}_{i j}:=\left\{k_{1}, \ldots k_{c_{i j}}\right\}$ is defined as the set of its $c_{i j}$ children. An illustrative example is presented in Figure 2: At level $i=1$ of the hierarchy, we have $\mathcal{E}_{1}=\{B, C\}$, and for element $C$ on that level we have $\mathcal{C}_{1 C}=\{F, G\}$. Similarly, $\mathcal{E}_{2}=\{D, E, F, G\}$. 


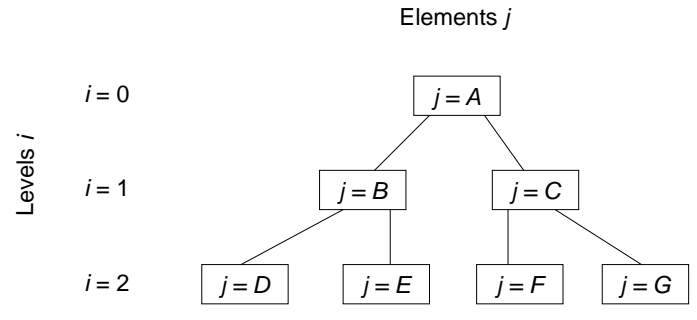

Figure 2: Example of index notation for a hierarchically partitioned design problem

- Elements at the same level of the hierarchy having the same parent element can share design variables called linking design variables $\mathbf{y}_{i j}$, for element $j$ at the $i$-th level. This concept can be extended to elements at the same level of the hierarchy having a common ancestor element.

- Responses $\mathbf{R}_{i}$ for $i$-level elements can be associated either with (top-level) supersystem targets $\mathbf{T}$ or with "cascaded down" or "passed up" targets $\mathbf{R}_{i}^{i-1}$ or $\mathbf{R}_{i}^{i+1}$, respectively. The latter quantities link two successive levels in the design hierarchy according to the ATC process.

- Responses $\mathbf{R}_{i j}$ of a system corresponding to element $j$ at the $i$-th level depend on responses $\mathbf{R}_{(i+1) k}, \quad k \in \mathcal{C}_{i j}$, of the subsystems making up the system, and on the system's local design variables $\mathbf{x}_{i j}$ and linking design variables $\mathbf{y}_{i j}$, i.e., $\quad \mathbf{R}_{i j}=$ $\mathbf{r}_{i j}\left(\mathbf{R}_{(i+1) k_{1}}, \ldots, \mathbf{R}_{(i+1) k_{c_{i j}}}, \mathbf{x}_{i j}, \mathbf{y}_{i j}\right)$. In general, the response or behavior of an element depends on design variables characterizing the element as well as on responses of (lower-level) elements making up the element.

- Element constraint functions $\mathbf{g}$ and $\mathbf{h}$ define the feasible space of the element's design variables and responses.

- Dummy elements can be introduced in the design hierarchy to account for cases in which element responses depend on responses of elements two or more levels down in the hierarchy.

- A tree is a connected graph without any circuits, and a forest is a collection of trees ${ }^{16}$. Note that a subgraph of a tree could be a forest. This is the case in Section 3, where the forests associated with design subproblems are derived from the hierarchy tree of the original problem.

\subsection{The design target problem}

In the context of ATC, the design target problem in (1) can be stated as follows: determine the values of design variables $\mathbf{x}$ that minimize the deviation of supersystem responses $\mathbf{R}$ from predefined targets $\mathbf{T}$ subject to design constraints. Under the assumption that supersystem responses depend on supersystem design variables and system responses, system responses depend on system design variables and subsystem responses, and so on down the design hierarchy, it can be concluded that supersystem responses and constraints depend on supersystem, system, subsystem and component design variables.

Assuming a hierarchical structure of the design target problem with $N+1$ levels, Problem (1) can be expressed as follows:

$$
\begin{aligned}
& \left.\min _{\left\{\mathbf{x}_{i j}, \mathbf{y}_{i j} \mid\right.} \| \mathcal{E}_{i}, i=0, \ldots, N\right\} \\
& \text { subject to } \\
& \mathbf{g}_{i j}\left(\mathbf{R}_{i j}, \mathbf{x}_{i j}, \mathbf{y}_{i j}\right) \leq \mathbf{T} \|, \quad l \in \mathcal{E}_{0} \\
& \mathbf{h}_{i j}\left(\mathbf{R}_{i j}, \mathbf{x}_{i j}, \mathbf{y}_{i j}\right)=\mathbf{0} \\
& \mathbf{R}_{i j}-\mathbf{r}_{i j}\left(\mathbf{R}_{(i+1) k_{1}}, \ldots, \mathbf{R}_{(i+1) k_{c_{i j}}}, \mathbf{x}_{i j}, \mathbf{y}_{i j}\right) \\
& =\mathbf{0}, \quad \forall j \in \mathcal{E}_{i}, i=0, \ldots, N,
\end{aligned}
$$

where for each element $j$ at the $i$-th level,

- $\mathbf{x}_{i j} \in \mathbb{R}^{n_{i j}}$ is the vector of $n_{i j}$ local design variables, that is, variables exclusively associated with the element;

- $\mathbf{y}_{i j} \in \mathbb{R}^{l_{i j}}$ is the vector of $l_{i j}$ linking design variables, that is, variables associated with the element and one or more other elements that share the same parent; compatibility among linking design variables is enforced by sharing components of the vectors $\mathbf{y}_{i j}$ between different elements $j$ that share the same parent;

- $\mathbf{R}_{i j} \in \mathbb{R}^{d_{i j}}$ is the vector of $d_{i j}$ responses;

- $\mathbf{r}_{i j}: \mathbb{R}^{a_{i j}} \rightarrow \mathbb{R}^{d_{i j}}$ are response vector functions, where $a_{i j}:=n_{i j}+l_{i j}+\sum_{k \in \mathcal{C}_{i j}} d_{(i+1) k}$;

- $\mathbf{g}_{i j}: \mathbb{R}^{b_{i j}} \rightarrow \mathbb{R}^{v_{i j}}$ and $\mathbf{h}_{i j}: \mathbb{R}^{b_{i j}} \rightarrow \mathbb{R}^{u_{i j}}$ are vector functions representing $v_{i j}$ inequality and $u_{i j}$ equality design constraints, respectively, where $b_{i j}:=d_{i j}+n_{i j}+l_{i j}$; and

- $\|\cdot\|$ is a norm; typically, a weighted norm is used for the targets $\mathbf{T}$ to enable trade-off evaluation studies, while the $l_{2}$-norm is used in all other cases.

Note that at the (top) 0-th level, there are no linking design variables $\mathbf{y}_{0 l}, l \in \mathcal{E}_{0}$. Moreover, 
at the (bottom) $N$-th level, element responses depend only on the element's design variables; thus, the last equality constraints in (2) become $\mathbf{R}_{N j}$ $\mathbf{r}_{N j}\left(\mathbf{x}_{N j}, \mathbf{y}_{N j}\right)=\mathbf{0}, \forall j \in \mathcal{E}_{N}$.

\subsection{Relaxation of the design target problem}

To study the convergence properties of ATC applied to the design target problem in (2), we relax the interactions between every two consecutive levels of the problem hierarchy by introducing local copies of the responses at each level. Namely, we introduce superscripts that denote the levels at which the individual responses are computed. Moreover, compatibility of linking design variables at a given level is enforced by introducing copies of these variables at the corresponding upper level. Hence, a relaxation of the problem in (2) is

$$
\begin{aligned}
& \min _{\left\{\overline{\mathbf{x}}_{i j}^{i} \mid\right.}\left\|\mathbf{R}_{0 l}^{0}-\mathbf{T}\right\|+\sum_{i=0}^{N-1} \sum_{\left.j \in \mathcal{E}_{i}, i=0, \ldots, N\right\}} \epsilon_{i j}^{R} \\
& +\sum_{i=0}^{N-1} \sum_{j \in \mathcal{E}_{i}} \epsilon_{i j}^{y}, \quad l \in \mathcal{E}_{0} \\
& \text { subject to } \\
& \mathbf{g}_{i j}\left(\mathbf{R}_{i j}^{i}, \mathbf{x}_{i j}^{i}, \mathbf{y}_{i j}^{i}\right) \leq \mathbf{0}, \\
& \mathbf{h}_{i j}\left(\mathbf{R}_{i j}^{i}, \mathbf{x}_{i j}^{i}, \mathbf{y}_{i j}^{i}\right)=\mathbf{0} \text {, } \\
& \mathbf{R}_{i j}^{i}-\mathbf{r}_{i j}\left(\mathbf{R}_{(i+1) k_{1}}^{i}, \ldots, \mathbf{R}_{(i+1) k_{c_{i j}}}^{i}, \mathbf{x}_{i j}^{i}, \mathbf{y}_{i j}^{i}\right) \\
& =\mathbf{0}, \forall j \in \mathcal{E}_{i}, i=0, \ldots, N \text {, } \\
& \sum_{k \in \mathcal{C}_{i j}} w_{(i+1) k}^{R}\left\|\mathbf{R}_{(i+1) k}^{i}-\mathbf{R}_{(i+1) k}^{i+1}\right\| \leq \epsilon_{i j}^{R}, \\
& \sum_{k \in \mathcal{C}_{i j}} w_{(i+1) k}^{y}\left\|\mathbf{y}_{(i+1) k}^{i}-\mathbf{y}_{(i+1) k}^{i+1}\right\| \leq \epsilon_{i j}^{y}, \\
& \forall j \in \mathcal{E}_{i}, i=0, \ldots, N-1 .
\end{aligned}
$$

In the above formulation for element $j$ at the $i$-th level and element $k$ at the $(i+1)$-th level,

$$
\begin{aligned}
\overline{\mathbf{x}}_{i j}^{i}:= & {\left[\mathbf{x}_{i j}^{i}, \mathbf{y}_{i j}^{i}, \mathbf{y}_{(i+1) k_{1}}^{i}, \ldots, \mathbf{y}_{(i+1) k_{c_{i j}}}^{i},\right.} \\
& \left.\mathbf{R}_{(i+1) k_{1}}^{i}, \ldots, \mathbf{R}_{(i+1) k_{c_{i j}}}^{i}, \epsilon_{i j}^{R}, \epsilon_{i j}^{y}\right]^{t}
\end{aligned}
$$

is the vector of optimization variables;

- $w_{(i+1) k}^{R} \in \mathbb{R}_{\geq 0}$ is the weighting coefficient for the deviation of responses $\mathbf{R}_{(i+1) k}$ computed at the $i$-th and $(i+1)$-th levels;

- $\epsilon_{i j}^{R} \in \mathbb{R}_{\geq 0}$ is the tolerance variable for compatibility of responses between the $i$-th and $(i+1)$-th levels;
- $w_{(i+1) k}^{y} \in \mathbb{R}_{\geq 0}$ is the weighting coefficient for the deviation of linking design variables $\mathbf{y}_{(i+1) k}$ computed at the $i$-th and $(i+1)$-th levels;

- $\epsilon_{i j}^{y} \in \mathbb{R}_{\geq 0}$ is the tolerance variable for compatibility of linking design variables at the $(i+1)$-th level;

- $\mathbf{R}_{i j}^{i} \in \mathbb{R}^{d_{i j}}$ is the $i$-th level copy of the vector of $d_{i j}$ responses;

- $\mathbf{R}_{(i+1) k}^{i} \in \mathbb{R}^{l_{(i+1) k}}, k \in \mathcal{C}_{i j}$, is the $i$-th level copy of the vector of $d_{(i+1) k}$ responses associated with the children of element $j$;

- $\mathbf{x}_{i j}^{i} \in \mathbb{R}^{n_{i j}}$ is the same vector as $\mathbf{x}_{i j}$, the vector of $n_{i j}$ local design variables; although there is only one copy of these variables, the superscript $i$ has been added for consistency with the linking design variables;

- $\mathbf{y}_{i j}^{i} \in \mathbb{R}^{l_{i j}}$ is the $i$-th level copy of the vector of $l_{i j}$ linking design variables, that is, variables associated with the element and one or more other elements that share the same parent; note that $\mathbf{y}_{i j}^{i}$ for $j \in \mathcal{E}_{i}$ are independent of each other, i.e., they do not share components;

- $\mathbf{y}_{(i+1) k}^{i} \in \mathbb{R}^{l_{(i+1) k}}, k \in \mathcal{C}_{i j}$, is the $i$-th level copy of the vector of $l_{(i+1) k}$ linking design variables associated with the children of element $j$; compatibility among linking design variables of the children of element $j$ is enforced by sharing components of the vectors $\mathbf{y}_{(i+1) k}^{i}$ between different elements $k \in \mathcal{C}_{i j}$.

Note again that at the (top) 0-th level, there are no linking design variables $\mathbf{y}_{0 l}^{0}, l \in \mathcal{E}_{0}$. Moreover, at the (bottom) $N$-th level, element responses depend only on the element's design variables; thus, the last equality constraints in (3) become $\mathbf{R}_{N j}^{N}-$ $\mathbf{r}_{N j}\left(\mathbf{x}_{N j}, \mathbf{y}_{N j}^{N}\right)=\mathbf{0}$, for $\forall j \in \mathcal{E}_{N}$. Also, $\epsilon_{i j}^{R}$ and $\epsilon_{i j}^{y}$ appear in the objective function to be minimized, and thus the inequality constraints involving $\epsilon_{i j}^{R}$ and $\epsilon_{i j}^{y}$ are active and can be included in the objective 
function as following:

$$
\begin{aligned}
& \min _{\left\{\overline{\mathbf{x}}_{i j}^{i} \mid\right.}\left\|\mathbf{R}_{\left.j \in \mathcal{E}_{i}, i=0, \ldots, N\right\}}^{0}-\mathbf{T}\right\|+ \\
& \sum_{i=0}^{N-1} \sum_{j \in \mathcal{E}_{i}} \sum_{k \in \mathcal{C}_{i j}} w_{(i+1) k}^{R}\left\|\mathbf{R}_{(i+1) k}^{i}-\mathbf{R}_{(i+1) k}^{i+1}\right\|+ \\
& \sum_{i=0}^{N-1} \sum_{j \in \mathcal{E}_{i}} \sum_{k \in \mathcal{C}_{i j}} w_{(i+1) k}^{y}\left\|\mathbf{y}_{(i+1) k}^{i}-\mathbf{y}_{(i+1) k}^{i+1}\right\|, \quad l \in \mathcal{E}_{0}
\end{aligned}
$$$$
\text { subject to }
$$$$
\mathbf{g}_{i j}\left(\mathbf{R}_{i j}^{i}, \mathbf{x}_{i j}^{i}, \mathbf{y}_{i j}^{i}\right) \leq \mathbf{0} \text {, }
$$$$
\mathbf{h}_{i j}\left(\mathbf{R}_{i j}^{i}, \mathbf{x}_{i j}^{i}, \mathbf{y}_{i j}^{i}\right)=\mathbf{0} \text {, }
$$$$
\mathbf{R}_{i j}^{i}-\mathbf{r}_{i j}\left(\mathbf{R}_{(i+1) k_{1}}^{i}, \ldots, \mathbf{R}_{(i+1) k_{c_{i j}}}^{i}, \mathbf{x}_{i j}^{i}, \mathbf{y}_{i j}^{i}\right)
$$$$
=\mathbf{0}, \forall j \in \mathcal{E}_{i}, i=0, \ldots, N \text {, }
$$

where

$$
\begin{aligned}
\overline{\mathbf{x}}_{i j}^{i}:= & {\left[\mathbf{x}_{i j}^{i}, \mathbf{y}_{i j}^{i}, \mathbf{y}_{(i+1) k_{1}}^{i}, \ldots, \mathbf{y}_{(i+1) k_{c_{i j}}}^{i},\right.} \\
& \left.\mathbf{R}_{(i+1) k_{1}}^{i}, \ldots, \mathbf{R}_{(i+1) k_{c_{i j}}}^{i}\right]^{t}
\end{aligned}
$$

is the vector of optimization variables of element $j$ at the $i$-th level.

\subsection{Description of the ATC process}

The analytical target cascading process solves a series of design target subproblems for each element in the design hierarchy, as shown in Figure 3 for a hypothetical four-level hierarchy. The design target subproblem at the supersystem level is to minimize the deviation between supersystem targets and associated responses subject to constraints for system responses, system linking design variables, and supersystem design constraints. Once system responses and linking design variables are determined by solving this subproblem, they are cascaded down to the system level as targets. Similar subproblems are formulated for each element at lower levels of the hierarchy. Optimal responses and linking design variables are also passed up as constraint parameters to upper-level subproblems. Figure 4 shows the information flow described above in term of responses, local and linking design variables for a bilevel hierarchy. The design subproblems, which are represented by rectangular boxes, are solved according to the formulation given in Section 2.5, Problem (5). Analysis models, which are represented by ovals, are used to compute responses according to $\mathbf{R}_{i j}=\mathbf{r}_{i j}\left(\mathbf{R}_{(i+1) k_{1}}, \ldots, \mathbf{R}_{(i+1) k_{c_{i j}}}, \mathbf{x}_{i j}, \mathbf{y}_{i j}\right)$.

The top-down and bottom-up, level-by-level sequential solution of design subproblems described above and depicted in Figures 3 and 4 is not the only

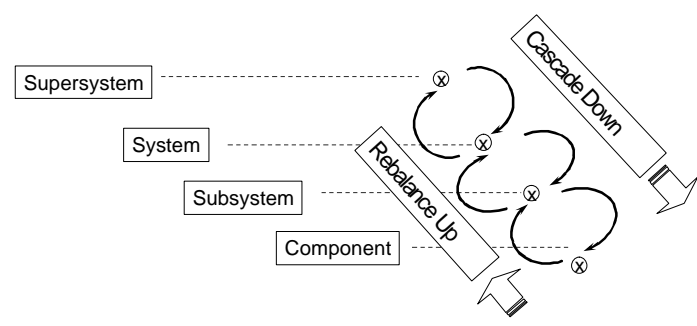

Figure 3: Schematic of the analytical target cascading process for a top-down and bottom-up, level-bylevel solution sequence

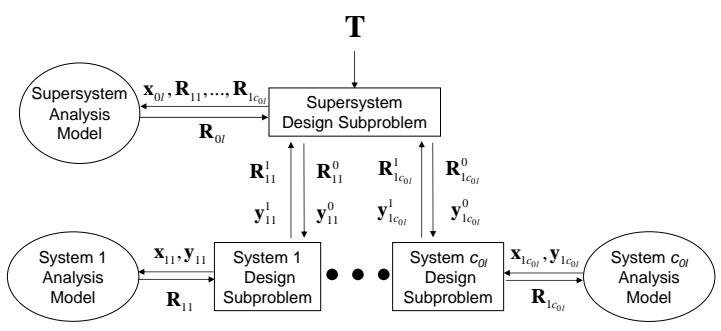

Figure 4: Information flow in analytical target cascading for a bilevel hierarchy

coordination sequence allowable in ATC. Other solution sequences can also be used to implement a convergent ATC process. As shown later in this article, an acceptable solution sequence should recursively divide the problem hierarchy into two subforests until the resulting subforests correspond to single levels of the original hierarchy. The separable subproblems for the elements of these single-level "forests" are then solved according to the general ATC formulation given in Section 2.5, Problems (5) and (6). Figure 5 shows four "convergent" solution sequences that can be used to implement ATC for a four-level design hierarchy. In Figure 5(a), supersystem and system subproblems are solved first; then subsystem and component subproblems are solved iteratively until some convergence criterion is achieved. This loop is then expanded to include system subproblems, and later the supersystem subproblem. In Figure 5(b), the supersystem subproblem is solved first; then system and subsystem subproblems are solved iteratively until convergence. This loop is expanded to include component subproblems, and later the supersystem subproblem. In Figure 5(c), supersystem and system subproblems are solved iteratively until convergence. Then subsystem and component subproblems are solved iteratively until convergence. Iteration continues between the supersystem/systems loop and the subsystems/components loop. The iterations in Figure 5(d) are analogous to those in 
Figure 5(b) after swapping supersystem and components, and systems and subsystems in the solution sequence. Similarly for the iteration sequence in Figure 5(e) with respect to that in Figure 5(a).

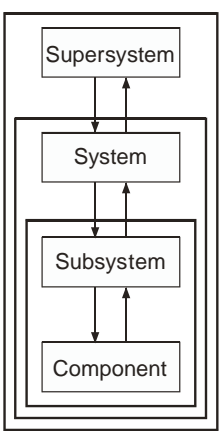

(a)

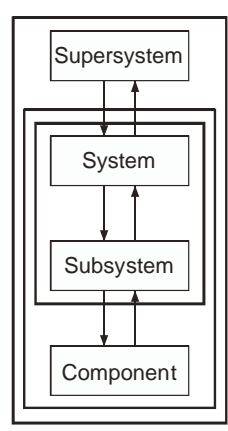

(b)

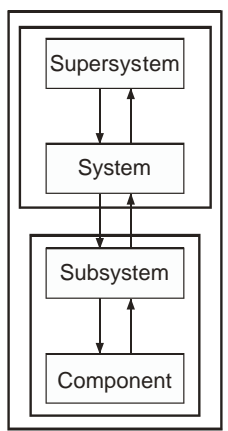

(c)

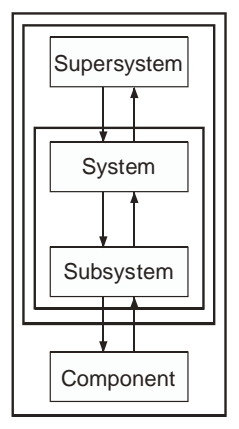

(d)

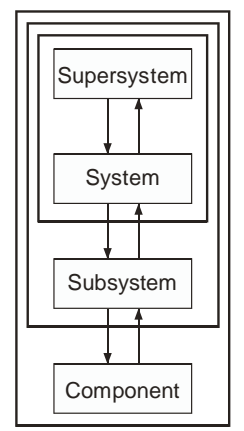

(e)
Figure 5: Schematics of analytical target cascading processes for various convergent solution sequences

\subsection{Formulation of ATC at a given level of the hi- erarchy}

The ATC design subproblem corresponding to the element $j$ at the $i$-th (intermediate) level is formulated as follows:

$$
\begin{gathered}
\min _{\overline{\mathbf{x}}_{i j}} w_{i j}^{R}\left\|\mathbf{R}_{i j}^{i}-\mathbf{R}_{i j}^{i-1}\right\|+w_{i j}^{y}\left\|\mathbf{y}_{i j}^{i}-\mathbf{y}_{i j}^{i-1}\right\|+ \\
\epsilon_{i j}^{R}+\epsilon_{i j}^{y}
\end{gathered}
$$

subject to

$$
\begin{aligned}
& \sum_{k \in \mathcal{C}_{i j}} w_{(i+1) k}^{R}\left\|\mathbf{R}_{(i+1) k}^{i}-\mathbf{R}_{(i+1) k}^{i+1}\right\| \leq \epsilon_{i j}^{R} \\
& \sum_{k \in \mathcal{C}_{i j}} w_{(i+1) k}^{y}\left\|\mathbf{y}_{(i+1) k}^{i}-\mathbf{y}_{(i+1) k}^{i+1}\right\| \leq \epsilon_{i j}^{y} \\
& \mathbf{g}_{i j}\left(\mathbf{R}_{i j}^{i}, \mathbf{x}_{i j}^{i}, \mathbf{y}_{i j}^{i}\right) \leq \mathbf{0} \\
& \mathbf{h}_{i j}\left(\mathbf{R}_{i j}^{i}, \mathbf{x}_{i j}^{i}, \mathbf{y}_{i j}^{i}\right)=\mathbf{0} \\
& \mathbf{R}_{i j}^{i}-\mathbf{r}_{i j}\left(\mathbf{R}_{(i+1) k_{1}}^{i}, \ldots, \mathbf{R}_{(i+1) k_{c_{i j}}}^{i}, \mathbf{x}_{i j}^{i}, \mathbf{y}_{i j}^{i}\right)=\mathbf{0}
\end{aligned}
$$

In the above formulation for the element $j$ at the $i$-th level,

$$
\begin{aligned}
\overline{\mathbf{x}}_{i j}:= & {\left[\mathbf{x}_{i j}^{i}, \mathbf{y}_{i j}^{i}, \mathbf{y}_{(i+1) k_{1}}^{i}, \ldots, \mathbf{y}_{(i+1) k_{c_{i j}}}^{i},\right.} \\
& \left.\mathbf{R}_{(i+1) k_{1}}^{i}, \ldots, \mathbf{R}_{(i+1) k_{c_{i j}}}^{i}, \epsilon_{i j}^{R}, \epsilon_{i j}^{y}\right]^{t}
\end{aligned}
$$

is the vector of optimization variables.

At the the top level, the term $\mathbf{R}_{0 l}^{-1}, l \in \mathcal{E}_{0}$, can be considered as the vector of supersystem targets $\mathbf{T}$, and the term $\left\|\mathbf{y}_{0 j}-\mathbf{y}_{0 j}^{-1}\right\|$ is missing. At the bottom level, the deviation constraints on responses and linking design variables are missing.

It is assumed for simplicity that all problems are continuous, but the formulation holds even if some optimization variables are discrete. In the latter case, suitable optimization algorithms are necessary for the solution of the associated mixed-integer programming problems.

Note again that $\epsilon_{i j}^{R}$ and $\epsilon_{i j}^{y}$ appear in the objective function to be minimized, and thus the two inequality constraints involving $\epsilon_{i j}^{R}$ and $\epsilon_{i j}^{y}$ are active and can be included in the objective function. Moreover, the subproblems in (5) for the elements at level $i$ are independent of each other, and can be combined into the following single problem by taking summation of the objective functions and union of the constraint functions.

$$
\begin{aligned}
& \min _{\left\{\overline{\mathbf{x}}_{i j}^{i} \mid j \in \mathcal{E}_{i}\right\}} \sum_{j \in \mathcal{E}_{i}} w_{i j}^{R}\left\|\mathbf{R}_{i j}^{i}-\mathbf{R}_{i j}^{i-1}\right\|+ \\
& \sum_{j \in \mathcal{E}_{i}} w_{i j}^{y}\left\|\mathbf{y}_{i j}^{i}-\mathbf{y}_{i j}^{i-1}\right\|+ \\
& \sum_{j \in \mathcal{E}_{i}} \sum_{k \in \mathcal{C}_{i j}} w_{(i+1) k}^{R}\left\|\mathbf{R}_{(i+1) k}^{i}-\mathbf{R}_{(i+1) k}^{i+1}\right\|+ \\
& \sum_{j \in \mathcal{E}_{i}} \sum_{k \in \mathcal{C}_{i j}} w_{(i+1) k}^{y}\left\|\mathbf{y}_{(i+1) k}^{i}-\mathbf{y}_{(i+1) k}^{i+1}\right\| \\
& \text { subject to } \\
& \mathbf{g}_{i j}\left(\mathbf{R}_{i j}^{i}, \mathbf{x}_{i j}^{i}, \mathbf{y}_{i j}^{i}\right) \leq \mathbf{0}, \\
& \mathbf{h}_{i j}\left(\mathbf{R}_{i j}^{i}, \mathbf{x}_{i j}^{i}, \mathbf{y}_{i j}^{i}\right)=\mathbf{0} \text {, } \\
& \mathbf{R}_{i j}^{i}-\mathbf{r}_{i j}\left(\mathbf{R}_{(i+1) k_{1}}^{i}, \ldots, \mathbf{R}_{(i+1) k_{c_{i j}}^{i}}, \mathbf{x}_{i j}^{i}, \mathbf{y}_{i j}^{i}\right) \\
& =\mathbf{0}, \forall j \in \mathcal{E}_{i} \text {. }
\end{aligned}
$$

The analytical target cascading (ATC) process solves the subproblems in (6) for all levels of the design hierarchy in an orderly and iterative fashion, converging to the solution of the original design target problem in (1) or (2). Some convergent solution sequences were shown in Figure 5. The main outcome of the ATC process is the final values of element responses, which represent the (cascaded) tar- 
gets for the systems, subsystems, and components of the supersystem.

\section{DESIGN TARGET SUBPROBLEM FOR A FOREST IN THE PROBLEM HIERARCHY}

In this section we define carefully the structure of the two adjacent subproblems that must be jointly solved iteratively in any ATC convergent solution strategy, such as those depicted in Figure 5. This will prepare us for the convergence arguments in Sections 4 and 5 .

Consider a forest $\mathbf{F}$ in the problem hierarchy covering all nodes and edges from level $i=p$ to level $i=r>p+1$. Figure 6 depicts such a general forest. Let $q$ be a number between $p$ and $r$. Decompose the

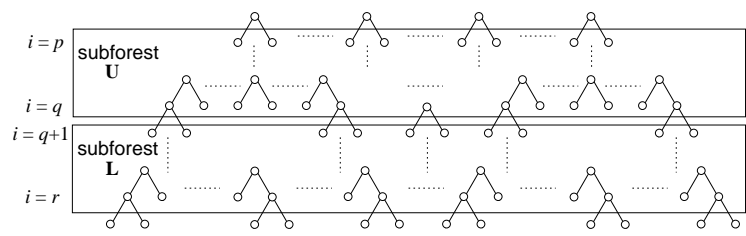

Figure 6: General forest in the problem hierarchy covering all nodes and edges from level $i=p$ to level $i=r$

forest $\mathbf{F}$ into two subforests $\mathbf{U}$ and $\mathbf{L}$. The subforest $\mathbf{U}$ consists of all the levels between $i=p$ and $i=q$, whereas the subforest $\mathbf{L}$ consists of all the levels between $i=q+1$ and $i=r$.

Then, from the overall problem in (4), the relaxed design target subproblem corresponding to the forest $\mathbf{F}$ can be formulated as in (7). This subproblem consists of all design constraints and responses between levels $p$ and $r$. Given that the subproblems for the subtrees rooted at the nodes at level $i=p$ (i.e., elements of $\mathcal{E}_{p}$ ) are independent of each other, they can be combined into a single problem by taking summation of their objective functions and union of their constraint functions.

$$
\begin{aligned}
& \min _{\left\{\overline{\mathbf{x}}_{i j}^{i} \mid\right.} \sum_{\left.j \in \mathcal{E}_{i}, i=p, \ldots, r\right\}}^{r} \sum_{i=p-1} \sum_{j \in \mathcal{E}_{i}} \sum_{k \in \mathcal{C}_{i j}} \\
& w_{(i+1) k}^{R}\left\|\mathbf{R}_{(i+1) k}^{i}-\mathbf{R}_{(i+1) k}^{i+1}\right\| \\
&+\sum_{i=p-1}^{r} \sum_{j \in \mathcal{E}_{i}} \sum_{k \in \mathcal{C}_{i j}} w_{(i+1) k}^{y}\left\|\mathbf{y}_{(i+1) k}^{i}-\mathbf{y}_{(i+1) k}^{i+1}\right\|
\end{aligned}
$$

subject to

$$
\begin{aligned}
& \mathbf{g}_{i j}\left(\mathbf{R}_{i j}^{i}, \mathbf{x}_{i j}^{i}, \mathbf{y}_{i j}^{i}\right) \leq \mathbf{0}, \\
& \mathbf{h}_{i j}\left(\mathbf{R}_{i j}^{i}, \mathbf{x}_{i j}^{i}, \mathbf{y}_{i j}^{i}\right)=\mathbf{0}, \\
& \mathbf{R}_{i j}^{i}-\mathbf{r}_{i j}\left(\mathbf{R}_{(i+1) k_{1}}^{i}, \ldots, \mathbf{R}_{(i+1) k_{c_{i j}}}^{i}, \mathbf{x}_{i j}^{i}, \mathbf{y}_{i j}^{i}\right) \\
& =\mathbf{0}, \quad \forall j \in \mathcal{E}_{i}, p \leq i \leq r .
\end{aligned}
$$

where

$$
\begin{aligned}
\overline{\mathbf{x}}_{i j}^{i}:= & {\left[\mathbf{x}_{i j}^{i}, \mathbf{y}_{i j}^{i}, \mathbf{y}_{(i+1) k_{1}}^{i}, \ldots, \mathbf{y}_{(i+1) k_{c_{i j}}}^{i},\right.} \\
& \left.\mathbf{R}_{(i+1) k_{1}}^{i}, \ldots, \mathbf{R}_{(i+1) k_{c_{i j}}}^{i}\right]^{t}
\end{aligned}
$$

is the vector of optimization variables of node $j$ at level $i$. Responses and linking design variables with superscripts $p-1$ and $r+1$ are fixed parameters cascaded down and passed up from upper and lower levels, respectively.

\subsection{Problem for the upper subforest}

The design target subproblem for the upper subforest $\mathbf{U}$ can be derived from the problem for $\mathbf{F}$ in (7) simply by replacing $r$ by $q$.

$$
\begin{aligned}
& \min _{\left\{\overline{\mathbf{x}}_{i j}^{i} \mid\right.} \sum_{\left.j \in \mathcal{E}_{i}, i=p, \ldots, q\right\}}^{q} \sum_{i=p-1} \sum_{j \in \mathcal{E}_{i}} \sum_{k \in \mathcal{C}_{i j}} \\
& w_{(i+1) k}^{R}\left\|\mathbf{R}_{(i+1) k}^{i}-\mathbf{R}_{(i+1) k}^{i+1}\right\| \\
& +\sum_{i=p-1}^{q} \sum_{j \in \mathcal{E}_{i}} \sum_{k \in \mathcal{C}_{i j}} w_{(i+1) k}^{y}\left\|\mathbf{y}_{(i+1) k}^{i}-\mathbf{y}_{(i+1) k}^{i+1}\right\|
\end{aligned}
$$

subject to

$\mathbf{g}_{i j}\left(\mathbf{R}_{i j}^{i}, \mathbf{x}_{i j}^{i}, \mathbf{y}_{i j}^{i}\right) \leq \mathbf{0}$

$\mathbf{h}_{i j}\left(\mathbf{R}_{i j}^{i}, \mathbf{x}_{i j}^{i}, \mathbf{y}_{i j}^{i}\right)=\mathbf{0}$,

$\mathbf{R}_{i j}^{i}-\mathbf{r}_{i j}\left(\mathbf{R}_{(i+1) k_{1}}^{i}, \ldots, \mathbf{R}_{(i+1) k_{c_{i j}}^{i}}, \mathbf{x}_{i j}^{i}, \mathbf{y}_{i j}^{i}\right)$

$=\mathbf{0}, \quad \forall j \in \mathcal{E}_{i}, p \leq i \leq q$.

where

$$
\begin{aligned}
\overline{\mathbf{x}}_{i j}^{i}:= & {\left[\mathbf{x}_{i j}^{i}, \mathbf{y}_{i j}^{i}, \mathbf{y}_{(i+1) k_{1}}^{i}, \ldots, \mathbf{y}_{(i+1) k_{c_{i j}}}^{i},\right.} \\
& \left.\mathbf{R}_{(i+1) k_{1}}^{i}, \ldots, \mathbf{R}_{(i+1) k_{c_{i j}}}^{i}\right]^{t}
\end{aligned}
$$

is the vector of optimization variables of node $j$ at level $i$. Responses and linking design variables with 
$p-1$ and $q+1$ superscripts are fixed parameters cascaded down and passed up from upper and lower levels, respectively.

\subsection{Problem for the lower subforest}

The design target subproblem for the lower subforest $\mathbf{L}$ can be derived from the problem for $\mathbf{F}$ in (7) simply by replacing $p-1$ by $q$.

$$
\begin{aligned}
& \min _{\left\{\overline{\mathbf{x}}_{i j}^{i} \mid j \in \mathcal{E}_{i}, i=q+1, \ldots, r\right\}} \sum_{i=q}^{r} \sum_{j \in \mathcal{E}_{i}} \sum_{k \in \mathcal{C}_{i j}} \\
& w_{(i+1) k}^{R}\left\|\mathbf{R}_{(i+1) k}^{i}-\mathbf{R}_{(i+1) k}^{i+1}\right\| \\
& +\sum_{i=q}^{r} \sum_{j \in \mathcal{E}_{i}} \sum_{k \in \mathcal{C}_{i j}} w_{(i+1) k}^{y}\left\|\mathbf{y}_{(i+1) k}^{i}-\mathbf{y}_{(i+1) k}^{i+1}\right\| \\
& \text { subject to } \\
& \mathbf{g}_{i j}\left(\mathbf{R}_{i j}^{i}, \mathbf{x}_{i j}^{i}, \mathbf{y}_{i j}^{i}\right) \leq \mathbf{0}, \\
& \mathbf{h}_{i j}\left(\mathbf{R}_{i j}^{i}, \mathbf{x}_{i j}^{i}, \mathbf{y}_{i j}^{i}\right)=\mathbf{0} \text {, } \\
& \mathbf{R}_{i j}^{i}-\mathbf{r}_{i j}\left(\mathbf{R}_{(i+1) k_{1}}^{i}, \ldots, \mathbf{R}_{(i+1) k_{c_{i j}}}^{i}, \mathbf{x}_{i j}^{i}, \mathbf{y}_{i j}^{i}\right) \\
& =\mathbf{0}, \quad \forall j \in \mathcal{E}_{i}, q+1 \leq i \leq r \text {. }
\end{aligned}
$$

where

$$
\begin{aligned}
\overline{\mathbf{x}}_{i j}^{i}:= & {\left[\mathbf{x}_{i j}^{i}, \mathbf{y}_{i j}^{i}, \mathbf{y}_{(i+1) k_{1}}^{i}, \ldots, \mathbf{y}_{(i+1) k_{c_{i j}}}^{i},\right.} \\
& \left.\mathbf{R}_{(i+1) k_{1}}^{i}, \ldots, \mathbf{R}_{(i+1) k_{c_{i j}}}^{i}\right]^{t}
\end{aligned}
$$

is the vector of optimization variables of node $j$ at level $i$. Responses and linking design variables with $q$ and $r+1$ superscripts are fixed parameters cascaded down and passed up from upper and lower levels, respectively.

\section{ATC AS PROBLEM COORDINATION BETWEEN TWO SUBFORESTS}

The problem for the forest $\mathbf{F}$ in (7) can be rewritten in the following simplified form:

$$
\min _{\mathbf{x}} f(\mathbf{x}) \text { subject to } \mathbf{g}(\mathbf{x}) \leq \mathbf{0} \text { and } \mathbf{h}(\mathbf{x})=\mathbf{0} \text {, }
$$

where $\mathbf{x} \in \mathbb{R}^{n}$ is the vector of all (independent and dependent) variables in Problem (7). Let $\mathbf{x}_{\mathbf{U}} \in \mathbb{R}^{n_{\mathbf{U}}}$ be the vector of all variables present in the "upper subforest problem" in (8) and $\mathbf{x}_{\mathbf{L}} \in \mathbb{R}^{n_{\mathbf{L}}}$ be the vector of all variables present in the "lower subforest problem" in (9), where $n=n_{\mathbf{U}}+n_{\mathbf{L}}$. Thus, by reordering variables if necessary, we have

$$
\mathrm{x}:=\left(\begin{array}{l}
\mathbf{x}_{\mathbf{U}} \\
\mathbf{x}_{\mathbf{L}}
\end{array}\right) .
$$

Note that $\mathbf{x}_{\mathbf{U}}$ consists of variables with superscripts between $p$ and $q$, and $\mathbf{x}_{\mathbf{L}}$ consists of variables with superscripts between $q+1$ and $r+1$.
Define $H_{\mathbf{U}}$ to be the submatrix of the identity matrix $I_{n}$ consisting of its first $n_{\mathbf{U}}$ rows, and $H_{\mathbf{L}}$ to be the submatrix of $I_{n}$ consisting of its last $n_{\mathbf{L}}$ rows. Then,

$$
\begin{aligned}
I_{n} & =\left(\begin{array}{l}
H_{\mathbf{U}} \\
H_{\mathbf{L}}
\end{array}\right) \\
H_{\mathbf{U}} \mathbf{x} & =\mathbf{x}_{\mathbf{U}} \\
H_{\mathbf{L}} \mathbf{x} & =\mathbf{x}_{\mathbf{L}} .
\end{aligned}
$$

The upper subforest problem in (8) and the lower subforest problem in (9) can be recovered by applying the hierarchical overlapping coordination (HOC) process ${ }^{17,18}$ to the combined problem in (7). That is, the problem in (8) can be recovered from the combined problem in (7) by fixing the quantities with superscripts between $q+1$ and $r+1$ as constants, whereas the problem in (9) can be recovered from the combined problem in (7) by fixing quantities with superscripts between $p-1$ and $q$ as constants. Moreover, quantities fixed to recover the upper subforest and lower subforest problems from the combined problem are determined from the solutions of the lower subforest and upper subforest problems, respectively.

The above coordination process, which corresponds to ATC applied to a forest $\mathbf{F}$ consisting of two subforests $\mathbf{U}$ and $\mathbf{L}$, can be rephrased using the simplified notation in (10) and (11): The variables $\mathbf{x}_{\mathbf{U}}$ in the upper subforest are first fixed at some feasible values, and then Problem (10) is solved. This determines the values of $\mathbf{x}_{\mathbf{L}}$. Problem (10) is solved again with these fixed values of $\mathbf{x}_{\mathbf{L}}$, which determines new values of $\mathbf{x}_{\mathbf{U}}$. This iterative process is repeated until a stable set of variable values are obtained, i.e., until the process converges.

One can easily prove that the above process converges $^{17,18}$ since, as shown in Section 5.1 below, the values of the objective function in (10) are decreasing during the iteration, and a monotonically decreasing sequence bounded below always converges. However, it is not certain that the accumulation point obtained by this process corresponds to the optimal solution of the combined problem in (10). This convergence will be addressed in Section 5.2.

The "passing-down" and "passing-up" ATC process can also be described in terms of the matrices $H_{\mathbf{U}}$ and $H_{\mathbf{L}}$ in (12). That is, for fixed and feasible values of the variables in the upper subforest, $\mathbf{x}_{\mathbf{U}}=\mathbf{d}_{\mathbf{U}}$, the passing-down problem is

$$
\begin{aligned}
& \min _{\mathbf{x}} f(\mathbf{x}) \text { subject to } \\
& \mathbf{g}(\mathbf{x}) \leq \mathbf{0}, \mathbf{h}(\mathbf{x})=\mathbf{0} \text { and } H_{\mathbf{U}} \mathbf{x}=\mathbf{d}_{\mathbf{U}},
\end{aligned}
$$


whereas for fixed and feasible values of the variables in the lower subforest, $\mathbf{x}_{\mathbf{L}}=\mathbf{d}_{\mathbf{L}}$, the passing-up problem is

$$
\begin{aligned}
& \min _{\mathbf{x}} f(\mathbf{x}) \text { subject to } \\
& \mathbf{g}(\mathbf{x}) \leq \mathbf{0}, \mathbf{h}(\mathbf{x})=\mathbf{0} \text { and } H_{\mathbf{L}} \mathbf{x}=\mathbf{d}_{\mathbf{L}}
\end{aligned}
$$

As stated earlier in this section, $\mathbf{d}_{\mathbf{U}}\left(\mathbf{d}_{\mathbf{L}}\right.$, resp.) is updated during the iterative ATC process by solving Problem (14) (Problem (13), resp.).

The following lemma states that the constraint vectors $\mathbf{g}$ and $\mathbf{h}$ in Problem (10) have a separable structure.

Lemma 4.1 The constraint vectors $\mathbf{g} \in \mathbb{R}^{P^{I}}$ and $\mathbf{h} \in \mathbb{R}^{p^{E}}$ in Problem (10), by reordering the constraints if necessary, have the following separable structure:

$$
\mathbf{g}=\left(\begin{array}{l}
\mathbf{g}_{\mathrm{U}} \\
\mathbf{g}_{\mathrm{L}}
\end{array}\right), \quad \mathbf{h}=\left(\begin{array}{c}
\mathbf{h}_{\mathrm{U}} \\
\mathbf{h}_{\mathrm{L}}
\end{array}\right)
$$

where $\mathbf{g}_{\mathbf{U}} \in \mathbb{R}^{p_{\mathbf{U}}^{I}}$ and $\mathbf{h}_{\mathbf{U}} \in \mathbb{R}^{p_{\mathbf{U}}^{E}}\left(\mathbf{g}_{\mathbf{L}}\right.$ and $\mathbf{h}_{\mathbf{L}}$, resp.) contain only variables $\mathbf{x}_{\mathbf{U}}\left(\mathbf{x}_{\mathbf{L}}\right.$, resp.).

Proof: Define $\mathbf{g}_{\mathbf{U}}, \mathbf{g}_{\mathbf{L}}, \mathbf{h}_{\mathbf{U}}$, and $\mathbf{h}_{\mathbf{L}}$ as following:

$$
\begin{aligned}
\mathbf{g}_{\mathbf{U}} & :=\left[\left\{\mathbf{g}_{i j} \mid j \in \mathcal{E}_{i}, p \leq i \leq q\right\}\right]^{t} \\
\mathbf{g}_{\mathbf{L}} & :=\left[\left\{\mathbf{g}_{i j} \mid j \in \mathcal{E}_{i}, q+1 \leq i \leq r\right\}\right]^{t} \\
\mathbf{h}_{\mathbf{U}} & :=\left[\left\{\mathbf{h}_{i j}, \mathbf{R}_{i j}^{i}-\mathbf{r}_{i j} \mid j \in \mathcal{E}_{i}, p \leq i \leq q\right\}\right]^{t} \\
\mathbf{h}_{\mathbf{L}} & :=\left[\left\{\mathbf{h}_{i j}, \mathbf{R}_{i j}^{i}-\mathbf{r}_{i j} \mid j \in \mathcal{E}_{i}, q+1 \leq i \leq r\right\}\right]^{t} .
\end{aligned}
$$

Dependency of constraint functions $\mathbf{g}_{\mathbf{U}}$ and $\mathbf{h}_{\mathbf{U}}$ ( $\mathbf{g}_{\mathbf{L}}$ and $\mathbf{h}_{\mathbf{L}}$, resp.) on variables $\mathbf{x}_{\mathbf{U}}\left(\mathbf{x}_{\mathbf{L}}\right.$, resp.) follows from constraint definitions in (8) and (9). That is, constraint functions $\mathbf{g}_{\mathbf{U}}$ and $\mathbf{h}_{\mathbf{U}}$ ( $\mathbf{g}_{\mathbf{L}}$ and $\mathbf{h}_{\mathbf{L}}$, resp.) depend on responses $\mathbf{R}_{i j}^{i}$, local design variables $\mathbf{x}_{i j}^{i}$ and linking design variables $\mathbf{y}_{i j}^{i}$ with superscripts $i$ between $p$ and $q(q+1$ and $r$, resp.).

The separable structure of the constraints in (7) plays a crucial role in proving the convergence of ATC since the objective function is also separable with respect to $\mathbf{x}_{\mathbf{U}}$ and $\mathbf{x}_{\mathbf{L}}$. That is, the solutions for Problem (8) and Problem (9) can be recovered from the solution for Problem (7).

Remark 4.2 The integer $p^{I}$ ( $p^{E}$, resp.) indicates the number of inequality (equality, resp.) constraints in Problem (10). The integer $p_{\mathbf{U}}^{I}\left(p_{\mathbf{U}}^{E}\right.$, resp.) is the number of inequality (equality, resp.) constraints involving variables $\mathbf{x}_{\mathbf{U}}$ only.

\section{Convergence of AnAlytical target CASCADING}

We are now ready to complete the convergence proof for the ATC coordination process. Essentially we justify why the coordination paths illustrated earlier in Figure 5 will actually lead to the solution of the original design target problem.

\subsection{Convergence to an accumulation point}

As mentioned in Section 3, the ATC process applied to a general forest $\mathbf{F}$ of the problem hierarchy corresponds to the hierarchical overlapping coordination (HOC) process described in ${ }^{17,18,19}$. The following properties have been observed in ${ }^{19}$ and show convergence of the ATC process to an accumulation point.

1. If the $\mathrm{HOC}$ algorithm is initiated with a feasible point $\mathbf{x}_{0}$, then at each stage of the process, the problems in (13) and (14) will have nonempty feasible domains.

2. If the sequences $\left\{\mathbf{x}_{\mathbf{L}_{i}}\right\}_{i=1}^{\infty}$ and $\left\{\mathbf{x}_{\mathbf{U}_{i}}\right\}_{i=1}^{\infty}$ result from solving Problem (13) and Problem (14), respectively, and $f^{\min }:=\min \{f(\mathbf{x}) \mid \mathbf{h}(\mathbf{x})=$ $\mathbf{0}, \mathbf{g}(\mathbf{x}) \leq \mathbf{0}\}$, then
(a) $f\left(\mathbf{x}_{\mathbf{U}_{i}}, \mathbf{x}_{\mathbf{L}_{i}}\right) \geq f\left(\mathbf{x}_{\mathbf{U}_{i}}, \mathbf{x}_{\mathbf{L}_{(i+1)}}\right) \geq$ $f\left(\mathbf{x}_{\mathbf{U}_{(i+1)}}, \mathbf{x}_{\mathbf{L}_{(i+1)}}\right)$
(b) $\lim _{i \rightarrow \infty} f\left(\mathbf{x}_{\mathbf{L}_{i}}\right)=\lim _{i \rightarrow \infty} f\left(\mathbf{x}_{\mathbf{U}_{i}}\right)=f^{*} \geq$ $f^{\min }$

3. Any accumulation point $\mathbf{x}^{*}$ of either $\left\{\mathbf{x}_{\mathbf{L}_{i}}\right\}_{i=1}^{\infty}$ or $\left\{\mathbf{x}_{\mathbf{U}_{i}}\right\}_{i=1}^{\infty}$ solves both Problem (13) and Problem (14).

\subsection{Convergence to the optimum of overall problem} Let $J(\mathbf{x})$ be the $\left(p^{I}+p^{E}\right) \times n$ matrix $\left(\begin{array}{c}J^{I}(\mathbf{x}) \\ J^{E}(\mathbf{x})\end{array}\right)$ where $J^{I}(\mathbf{x})$ and $J^{E}(\mathbf{x})$ are the Jacobians of $\mathbf{g}(\mathbf{x})$ and $\mathbf{h}(\mathbf{x})$, respectively. This matrix function $J(\mathbf{x})$ will be simply referred to as the Jacobian of Problem (10).

For a fixed point $\mathbf{x}^{*} \in \mathbb{R}^{n}$, define $T_{a}\left(\mathbf{x}^{*}\right)$ to be the set of the indices corresponding to the active inequality constraints at $\mathbf{x}^{*}$, i.e.,

$$
T_{a}\left(\mathbf{x}^{*}\right):=\left\{i \mid g_{i}\left(\mathbf{x}^{*}\right)=0\right\},
$$

where $g_{i}$ denotes the $i$-th inequality constraint.

Define an integer $p_{a}^{I}$ to be the number of active inequality constraints at $\mathbf{x}^{*}$. The integer $p_{a, \mathbf{U}}^{I}\left(p_{a, \mathbf{L}}^{I}\right.$, resp.) is the number of active inequality constraints involving variables $\mathbf{x}_{\mathbf{U}}\left(\mathbf{x}_{\mathbf{L}}\right.$, resp.) only. Note that Lemma 4.1 implies $p_{a}^{I}=p_{a, \mathbf{U}}^{I}+p_{a, \mathbf{L}}^{I}$. Let a $p_{a}^{I} \times n$ matrix $J_{a}^{I}\left(\mathbf{x}^{*}\right)$ be the submatrix of $J^{I}\left(\mathbf{x}^{*}\right)$ consisting of the active inequality constraints at $\mathrm{x}^{*}$. 
The Lagrange multiplier theorem ${ }^{20}$ states that a regular point $\mathbf{x}^{*} \in \mathbb{R}^{n}$ is a solution to Problem (10) if and only if there exists a nonnegative vector $\boldsymbol{\lambda}^{I} \in$ $\mathbb{R}^{p_{a}^{I}}$ and a vector $\boldsymbol{\lambda}^{E} \in \mathbb{R}^{p^{E}}$ such that

$$
\nabla f^{t}\left(\mathbf{x}^{*}\right)+J_{a}^{I}\left(\mathbf{x}^{*}\right)^{t} \boldsymbol{\lambda}^{I}+J^{E}\left(\mathbf{x}^{*}\right)^{t} \boldsymbol{\lambda}^{E}=\mathbf{0} .
$$

Condition (15) is equivalent to

$$
-\nabla f^{t}\left(\mathbf{x}^{*}\right)=J_{a}^{I}\left(\mathbf{x}^{*}\right)^{t} \boldsymbol{\lambda}^{I}+J^{E}\left(\mathbf{x}^{*}\right)^{t} \boldsymbol{\lambda}^{E}, \boldsymbol{\lambda}^{I} \geq \mathbf{0} .
$$

Let $\mathbf{x}^{*}$ be an accumulation point of the ATC process for a general forest $\mathbf{F}$. Since it is a solution of both Problem (13) and Problem (14), there exist vectors $\mathbf{z}^{I} \geq \mathbf{0}, \mathbf{z}^{E}, \mathbf{u}$ and vectors $\mathbf{w}^{I} \geq \mathbf{0}, \mathbf{w}^{E}, \mathbf{v}$ such that the following two equalities simultaneously hold.

$$
\begin{gathered}
-\nabla f^{t}\left(\mathbf{x}^{*}\right)=J_{a}^{I}\left(\mathbf{x}^{*}\right)^{t} \mathbf{z}^{I}+J^{E}\left(\mathbf{x}^{*}\right)^{t} \mathbf{z}^{E}+H_{\mathbf{U}}^{t} \mathbf{u} \\
-\nabla f^{t}\left(\mathbf{x}^{*}\right)=J_{a}^{I}\left(\mathbf{x}^{*}\right)^{t} \mathbf{w}^{I}+J^{E}\left(\mathbf{x}^{*}\right)^{t} \mathbf{w}^{E}+H_{\mathbf{L}}^{t} \mathbf{v}
\end{gathered}
$$

Therefore, the convergence of the ATC process for a general forest $\mathbf{F}$ boils down to the following question:

If $\mathbf{x}^{*}$ satisfies both equations in (17), does it automatically satisfy Eq. (16) for some vectors $\boldsymbol{\lambda}^{I} \geq \mathbf{0}$ and $\boldsymbol{\lambda}^{E}$ ?

The answer to this question is "yes", mainly due to the separable structure of the constraint functions shown in Lemma 4.1.

Theorem 5.1 If $\mathrm{x}^{*}$ is a solution to both Problem (13) and Problem (14), then it is a solution to Problem (10). That is, if $\mathbf{x}^{*}$ is a solution to both Problem (8) and Problem (9), then it is a solution to Problem (7).

Proof: Let $\mathbf{x}^{*}$ be a solution to both Problem (13) and Problem (14). Then, there exist vectors

$\mathbf{z}^{I}=\left(\begin{array}{c}z_{1}^{I} \\ \vdots \\ z_{p_{a}^{I}}^{I}\end{array}\right) \geq \mathbf{0}, \quad \mathbf{z}^{E}=\left(\begin{array}{c}z_{1}^{E} \\ \vdots \\ z_{p^{E}}^{E}\end{array}\right), \quad \mathbf{u}=\left(\begin{array}{c}u_{1} \\ \vdots \\ u_{n_{\mathbf{U}}}\end{array}\right)$,

and the vectors

$\mathbf{w}^{I}=\left(\begin{array}{c}w_{1}^{I} \\ \vdots \\ w_{p_{a}^{I}}^{I}\end{array}\right) \geq \mathbf{0}, \quad \mathbf{w}^{E}=\left(\begin{array}{c}w_{1}^{E} \\ \vdots \\ w_{p^{E}}^{E}\end{array}\right), \quad \mathbf{v}=\left(\begin{array}{c}v_{1} \\ \vdots \\ v_{n_{\mathbf{L}}}\end{array}\right)$,

such that both equations in (17) simultaneously hold. Define a nonnegative vector $\lambda^{I} \in \mathbb{R}^{p_{a}^{I}}$ and a vector $\boldsymbol{\lambda}^{E} \in \mathbb{R}^{p^{E}}$ as follows:

$$
\boldsymbol{\lambda}^{I}=\left(\begin{array}{c}
w_{1}^{I} \\
\vdots \\
w_{p_{a, \mathbf{U}}^{I}}^{I} \\
z_{p_{a, \mathbf{U}}^{I}+1}^{I} \\
\vdots \\
z_{p_{a}^{I}}^{I}
\end{array}\right), \quad \boldsymbol{\lambda}^{E}=\left(\begin{array}{c}
w_{1}^{E} \\
\vdots \\
w_{p_{\mathbf{U}}^{E}}^{E} \\
z_{p_{\mathbf{U}}^{E}+1} \\
\vdots \\
z_{p^{E}}^{E}
\end{array}\right)
$$

Recall that the integer $p_{a, \mathbf{U}}^{I}\left(p_{\mathbf{U}}^{E}\right.$, resp. $)$ is the number of active inequality constraints (equality constraints, resp.) involving variables $\mathbf{x}_{\mathbf{U}}$ only. We claim that these $\boldsymbol{\lambda}^{I}$ and $\boldsymbol{\lambda}^{E}$ satisfy Eq. (16), which implies that $\mathbf{x}^{*}$ is a solution to Problem (10).

To show the claim, define the matrices $A_{\mathbf{U}}^{I}, A_{\mathbf{L}}^{I}$, $A_{\mathbf{U}}^{E}$ and $A_{\mathbf{L}}^{E}$ as follows:

$$
\begin{aligned}
& J_{a}^{I}\left(\mathbf{x}^{*}\right)=\left(A_{\mathbf{U}}^{I}, A_{\mathbf{L}}^{I}\right) \\
& J^{E}\left(\mathbf{x}^{*}\right)=\left(A_{\mathbf{U}}^{E}, A_{\mathbf{L}}^{E}\right)
\end{aligned}
$$

where $A_{\mathbf{U}}^{I}$ and $A_{\mathbf{U}}^{E}$ are the first $n_{\mathbf{U}}$ columns of the matrices $J_{a}^{I}\left(\mathbf{x}^{*}\right)$ and $J^{E}\left(\mathbf{x}^{*}\right)$, respectively.

The two equations in (17) can be rewritten as

$$
\begin{aligned}
-\nabla f^{t}\left(\mathbf{x}^{*}\right) & =\left(\begin{array}{c}
A_{\mathbf{U}}^{I}{ }^{t} \\
A_{\mathbf{L}}^{I}
\end{array}\right) \mathbf{z}^{I}+\left(\begin{array}{c}
A_{\mathbf{U}}^{E^{t}} \\
A_{\mathbf{L}}^{E^{t}}
\end{array}\right) \mathbf{z}^{E}+H_{\mathbf{U}}^{t} \mathbf{u} \\
& =\left(\begin{array}{c}
A_{\mathbf{U}}^{I}{ }^{t} \\
A_{\mathbf{L}}^{I}
\end{array}\right) \mathbf{w}^{I}+\left(\begin{array}{c}
A_{\mathbf{U}}^{E^{t}} \\
A_{\mathbf{L}}^{E^{t}}
\end{array}\right) \mathbf{w}^{E}+H_{\mathbf{L}}^{t} \mathbf{v}
\end{aligned}
$$

Since $\left(\begin{array}{l}H_{\mathbf{U}} \\ H_{\mathbf{L}}\end{array}\right)=I_{n}$, one gets

$$
\begin{aligned}
& \left(\begin{array}{c}
A_{\mathbf{U}}^{I} \\
A_{\mathbf{L}}^{I}
\end{array}\right)\left(\mathbf{z}^{I}-\mathbf{w}^{I}\right)+\left(\begin{array}{c}
A_{\mathbf{U}}^{E^{t}} \\
A_{\mathbf{L}}^{E^{t}}
\end{array}\right)\left(\mathbf{z}^{E}-\mathbf{w}^{E}\right) \\
= & H_{\mathbf{L}}^{t} \mathbf{v}-H_{\mathbf{U}}^{t} \mathbf{u} \\
= & \left(H_{\mathbf{U}}^{t}, H_{\mathbf{L}}^{t}\right)\left(\begin{array}{c}
-\mathbf{u} \\
\mathbf{v}
\end{array}\right) \\
= & \left(\begin{array}{c}
-\mathbf{u} \\
\mathbf{v}
\end{array}\right) .
\end{aligned}
$$

Therefore,

$$
\begin{aligned}
\mathbf{u} & =-A_{\mathbf{U}}^{I}{ }^{t}\left(\mathbf{z}^{I}-\mathbf{w}^{I}\right)-A_{\mathbf{U}}^{E^{t}}\left(\mathbf{z}^{E}-\mathbf{w}^{E}\right) \\
\mathbf{v} & =A_{\mathbf{L}}^{I^{t}}\left(\mathbf{z}^{I}-\mathbf{w}^{I}\right)+A_{\mathbf{L}}^{E^{t}}\left(\mathbf{z}^{E}-\mathbf{w}^{E}\right) .
\end{aligned}
$$


Hence,

$$
\begin{aligned}
& -\nabla f^{t}\left(\mathbf{x}^{*}\right) \\
& =\left(\begin{array}{c}
A_{\mathbf{U}}^{I t} \\
A_{\mathbf{L}}^{I^{t}}
\end{array}\right) \mathbf{z}^{I}+\left(\begin{array}{c}
A_{\mathbf{U}}^{E^{t}} \\
A_{\mathbf{L}}^{E^{t}}
\end{array}\right) \mathbf{z}^{E}+H_{\mathbf{U}}^{t} \mathbf{u} \\
& =\left(H_{\mathbf{U}}^{t}, H_{\mathbf{L}}^{t}\right)\left(\begin{array}{c}
A_{\mathbf{U}}^{I^{t}} \\
A_{\mathbf{L}}^{I^{t}}
\end{array}\right) \mathbf{z}^{I}+\left(H_{\mathbf{U}}^{t}, H_{\mathbf{L}}^{t}\right)\left(\begin{array}{c}
A_{\mathbf{U}}^{E^{t}} \\
A_{\mathbf{L}}^{E^{t}}
\end{array}\right) \mathbf{z}^{E}- \\
& H_{\mathbf{U}}^{t}\left[A_{\mathbf{U}}^{I}{ }^{t}\left(\mathbf{z}^{I}-\mathbf{w}^{I}\right)+A_{\mathbf{U}}^{E}{ }^{t}\left(\mathbf{z}^{E}-\mathbf{w}^{E}\right)\right] \\
& =H_{\mathbf{L}}^{t} A_{\mathbf{L}}^{I^{t}} \mathbf{z}^{I}+H_{\mathbf{L}}^{t} A_{\mathbf{L}}^{E^{t}} \mathbf{z}^{E}+H_{\mathbf{U}}^{t} A_{\mathbf{U}}^{I t} \mathbf{w}^{I}+ \\
& H_{\mathbf{U}}^{t} A_{\mathbf{U}}^{E^{t} \mathbf{w}^{E}} \\
& =\left(H_{\mathbf{U}}^{t}, H_{\mathbf{L}}^{t}\right)\left(\begin{array}{c}
A_{\mathbf{U}}^{I}{ }^{t} \mathbf{w}^{I} \\
A_{\mathbf{L}}^{I}{ }^{t} \mathbf{z}^{I}
\end{array}\right)+\left(H_{\mathbf{U}}^{t}, H_{\mathbf{L}}^{t}\right)\left(\begin{array}{c}
A_{\mathbf{U}}^{E^{t} \mathbf{w}^{E}} \\
A_{\mathbf{L}}^{E^{t}} \mathbf{z}^{E}
\end{array}\right) \\
& =\left(\begin{array}{c}
A_{\mathbf{U}}^{I}{ }^{t} \mathbf{w}^{I} \\
A_{\mathbf{L}}^{I^{t}} \mathbf{z}^{I}
\end{array}\right)+\left(\begin{array}{c}
A_{\mathbf{U}}^{E}{ }^{t} \mathbf{w}^{E} \\
A_{\mathbf{L}}^{E^{t}} \mathbf{z}^{E}
\end{array}\right) \text {. }
\end{aligned}
$$

By Theorem 4.1, the matrices $J_{a}^{I}\left(\mathbf{x}^{*}\right)$ and $J^{E}\left(\mathbf{x}^{*}\right)$ have the following block structure:

$$
\begin{aligned}
& J_{a}^{I}\left(\mathbf{x}^{*}\right)=\left(A_{\mathbf{U}}^{I}, A_{\mathbf{L}}^{I}\right)=\left(\begin{array}{cc}
\hat{A}_{\mathbf{U}}^{I} & 0 \\
0 & \hat{A}_{\mathbf{L}}^{I}
\end{array}\right) \\
& J^{E}\left(\mathbf{x}^{*}\right)=\left(A_{\mathbf{U}}^{E}, A_{\mathbf{L}}^{E}\right)=\left(\begin{array}{cc}
\hat{A}_{\mathbf{U}}^{E} & 0 \\
0 & \hat{A}_{\mathbf{L}}^{E}
\end{array}\right)
\end{aligned}
$$

This implies the block structure $A_{\mathbf{U}}^{I}=\left(\begin{array}{c}\hat{A}_{\mathbf{U}}^{I} \\ 0\end{array}\right), A_{\mathbf{L}}^{I}=$ $\left(\begin{array}{c}0 \\ \hat{A}_{\mathbf{L}}^{I}\end{array}\right), A_{\mathbf{U}}^{E}=\left(\begin{array}{c}\hat{A}_{\mathbf{U}}^{E} \\ 0\end{array}\right)$ and $A_{\mathbf{L}}^{E}=\left(\begin{array}{c}0 \\ \hat{A}_{\mathbf{L}}^{E}\end{array}\right)$. Using this block structure and the definitions of $\boldsymbol{\lambda}^{I}$ and $\boldsymbol{\lambda}^{E}$ in (18), one checks easily that

$$
\begin{aligned}
& \left(\begin{array}{l}
A_{\mathbf{U}}^{I}{ }^{t} \mathbf{w}^{I} \\
A_{\mathbf{L}}^{I} \mathbf{z}^{I}
\end{array}\right)=\left(\begin{array}{c}
A_{\mathbf{U}}^{I}{ }^{t} \\
A_{\mathbf{L}}^{I}
\end{array}\right) \boldsymbol{\lambda}^{I} \\
& \left(\begin{array}{c}
A_{\mathbf{U}}^{E^{t}} \mathbf{w}^{E} \\
A_{\mathbf{L}}^{E} \mathbf{z}^{E}
\end{array}\right)=\left(\begin{array}{c}
A_{\mathbf{U}}^{E}{ }^{t} \\
A_{\mathbf{L}}^{E}
\end{array}\right) \boldsymbol{\lambda}^{E} .
\end{aligned}
$$

Combining the above equalities with Eq. (19), one gets

$$
\begin{aligned}
-\nabla f^{t}\left(\mathbf{x}^{*}\right) & =\left(\begin{array}{c}
A_{\mathbf{U}}^{I} \\
A_{\mathbf{L}}^{I}
\end{array}\right) \boldsymbol{\lambda}^{I}+\left(\begin{array}{c}
A_{\mathbf{U}}^{E t} \\
A_{\mathbf{L}}^{E t}
\end{array}\right) \boldsymbol{\lambda}^{E} \\
& =J_{a}^{I}\left(\mathbf{x}^{*}\right)^{t} \boldsymbol{\lambda}^{I}+J^{E}\left(\mathbf{x}^{*}\right)^{t} \boldsymbol{\lambda}^{E}, \boldsymbol{\lambda}^{I} \geq \mathbf{0},
\end{aligned}
$$

which shows that $\mathbf{x}^{*}$ is indeed a solution of Problem (10).

\subsection{Convergence of ATC to the optimum of the original design target problem}

Consider a general forest $\mathbf{F}$ in the problem hierarchy covering all nodes and edges from level $i=p$ to level $i=r$. Decompose $\mathbf{F}$ into two subforests, and apply ATC coordination to $\mathbf{F}$ using this structure. It was shown in the previous section that the ATC coordination process produces the optimum solution of the relaxed design target problem for $\mathbf{F}$. Note that each subforest can be further decomposed into smaller subforests, and the same ATC process can be recursively applied to those subforests.

When $p=0$ and $r=N$, forest $\mathbf{F}$ becomes the hierarchy of the relaxed design target problems in (3) and (4). Given that consistency and feasibility are assumed for the original design target problem, it is possible to find weights $w_{(i+1) k}^{R}$ and $w_{(i+1) k}^{y}$ such that $\epsilon_{(i+1) k}^{R}$ and $\epsilon_{(i+1) k}^{y}$ in (3) converge to zero. Thus, the ATC process recursively applied to the problem hierarchy produces the optimum solution of the original design target problems in (1) and (2).

\section{Conclusion}

The ATC problem formulation possesses a fortuitous structure that enables a convergent behavior of several coordination strategies, patterned after the earlier strategy of hierarchical overlapping coordination. The main characteristic of the ATC convergent coordination strategies is the recursive solution of two "overlapping" problems at a time, as illustrated in Figure 5. Our computational experiences to date with several realistic case studies cited in the introduction support these theoretical findings. Moreover, actual convergence has not presented any excessive computational burden. In fact, our experiences show that the convergence criteria for the inner loops in Figure 5 can be initially relaxed and then tightened as the ATC process progresses. Complete relaxation of the inner-loop convergence tolerances results in the top-down and bottom-up, level-by-level solution sequence depicted in Figure 3, which has shown to be convergence for most of our applications.

\section{ACKNOWLEDGEMENT}

This work has been partially supported by the Automotive Research Center, a U. S. Army Center of Excellence for Modeling and Simulation of Ground Vehicles at the University of Michigan, and by a grant by Ford Motor Company. This support is gratefully acknowledged.

\section{REFERENCES}

${ }^{1}$ Kim, H. M., Target Cascading in Optimal System Design, Ph.D. thesis, University of Michigan, 2001.

${ }^{2}$ Kim, H. M., Michelena, N., Papalambros, P. Y., 
and Jiang, T., "Target Cascading in Optimal System Design," Proceedings 26th Design Automation Conference, Baltimore, MD, September 2000, Paper No. DETC2000/DAC-14265.

${ }^{3}$ Michelena, N., Kim, H. M., and Papalambros, P. Y., "A System Partitioning and Optimization Approach to Target Cascading," Proceedings of the 12th International Conference on Engineering Design, Vol. 2, Munich, August 1999, pp. 1109-1112.

${ }^{4}$ Kim, H. M., Rideout, D. G., Papalambros, P. Y., and Stein, J. L., "Analytical Target Cascading in Automotive Vehicle Design," Proceedings 27th Design Automation Conference, Pittsburgh, PA, September 2001, Paper No. DETC2001/DAC21079.

${ }^{5}$ Michelena, N., Loucas, L., Kokkolareas, M., Lin, C., Jung, D., Filipi, Z., Assanis, D., Papalambros, P., Peng, H., Stein, J., and Feury, M., "Design of an advanced heavy tactical truck: A target cascading case study," SAE International Truck and Bus Meeting and Exhibition, Chicago, IL, November 2001, Paper No. F2001-01-2793.

${ }^{6}$ Kim, H. M., Kokkolaras, M., Louca, L., Delagrammatikas, G., Michelena, N., Filipi, Z., Papalambros, P., and Assanis, D., "Target cascading in vehicle redesign: A class VI truck study," International Journal of Vehicle Design, Vol. 29, No. 3, 2002, pp. 199-225.

${ }^{7}$ Kokkolaras, M., Fellini, R., Kim, H. M., Michelena, N., and Papalambros, P., "Extension of the target cascading formulation to the design of product families," Structural and Multidisciplinary Optimization, 2002, To appear.

${ }^{8}$ Kirsch, U., Optimum Structural Design: Concepts, Methods, and Applications, McGraw-Hill, NY, 1981.

${ }^{9}$ Sobieszczanski-Sobieski, J., "A linear optimization method for large optimization problemsBlueprint for development," Technical memorandum TM-83242, NASA Langley Research Center, Hampton, VA, February 1982.

${ }^{10}$ Sobieszczanski-Sobieski, J., James, B., and Dovi, A., "Structural optimization by multilevel decomposition," Proceedings Structures, Structural Dynamics, and Materials Conference, AIAA/ASME/ASCE/AHS, 1983.

${ }^{11}$ Sobieszczanski-Sobieski, J., "Two alternative ways for solving the coordination problem in multilevel optimization," Structural Optimization, Vol. 6, 1993, pp. 205-215.

${ }^{12}$ Thareja, R. and Haftka, R., "Efficient singlelevel solution of hierarchical problems in structural optimization," AIAA Journal, Vol. 28, No. 3, March 1990, pp. 506-514.

${ }^{13}$ Vanderplaats, G., Yang, Y., and Kim, D., "Sequential linearization method for multilevel optimization," AIAA Journal, Vol. 28, No. 2, February 1990, pp. 290-295.

${ }^{14}$ Braun, R., Collaborative optimization: An architecture for large-scale distributed design, Ph.D. thesis, Stanford University, CA, 1996.

${ }^{15}$ Alexandrov, N. and Lewis, R., "Analytical and computational aspects of collaborative optimization," Technical Memorandum TM-2000-210104, NASA Langley Research Center, Hampton, VA, April 2000.

${ }^{16}$ Deo, N., Graph theory with applications to engineer and computer science, Prentice Hall, 1995.

${ }^{17}$ Michelena, N., Papalambros, P. Y., Park, H., and Kulkarni, D., "Hierarchical Overlapping Coordination for Large-Scale Optimization by Decomposition," AIAA Journal, Vol. 37, July 1999, pp. 890896.

${ }^{18}$ Park, H., Michelena, N., Kulkarni, D., and Papalambros, P., "Convergence criteria for hierarchical overlapping coordination under linear constraints," Computational Optimization and Applications, Vol. 18, 2001, pp. 273-293.

${ }^{19}$ Macko, D. and Haimes, Y., "Overlapping coordination of hierarchical structures," IEEE Transactions on Systems, Man, and Cybernetics, Vol. SMC8, 1978, pp. 745-751.

${ }^{20}$ Bertsekas, D., Nonlinear Programming, Athena Scientific, Belmont, MA, 1995. 